\title{
Scan-Conversion Algorithm for Ridge Point Detection on Tubular Objects
}

\author{
Sukmoon Chang ${ }^{1}$, Dimitris N. Metaxas ${ }^{1}$, and Leon Axel ${ }^{2}$ \\ 1 CBIM, Rutgers University, Piscataway, NJ, USA \\ \{sukmoon, dnm\}@cs.rutgers.edu \\ 2 Department of Radiology, New York University, New York, NY, USA \\ leon. axel@med.nyu.edu
}

\begin{abstract}
Anatomical structures contain various types of curvilinear or tube-like structures such as blood vessels and bronchial trees. In medical imaging, the extraction and representation of such structures are of clinical importance. Complex curvilinear structures can be best represented by their center lines (or skeletons) along their elongated direction. In this paper, a gradient-based method for ridge point extraction on tubular objects is presented. Using the gradients of distance maps or intensity profiles usually generates skeleton surfaces for $3 \mathrm{D}$ objects, which is not desirable for representing tubular objects. To extract only the points on the centerline, we first employ the gradient vector flow (GVF) technique and then apply eigenanalysis of the Hessian matrix to remove false positive points. We present various results of the method using CLSM (Confocal Laser Scanning Microscopy) images of blood fibrins and CT images of a skull and lungs. Our method is efficient and allows for completely automatic extraction of points along the centerline of a tubular object in its elongated direction.
\end{abstract}

\section{Introduction}

Anatomical structures contain various types of curvilinear or tube-like structures such as blood vessels and bronchial trees. In medical imaging, the extraction and representation of such objects are of clinical importance. Complex curvilinear structures can be best represented by their centerlines (or skeletons) along their elongated direction. Clinical applications of skeletons, for example, include radiation therapy planning, epilepsy surgical planning, and path planning for endoscopic navigation systems. Although a number of methods have been developed to extract centerlines of tubular objects, most of them are semi-automatic, requiring user intervention such as selecting seed points. In this paper, we present an efficient method for ridge point detection on tubular objects. This method allows for completely automatic extraction of the points along the centerline of a tubular object. We begin with the discussion of relevant methods for skeletonization. We then present our scan-conversion method in 2D and extend it to 3D. The skeletons of 3D objects normally consist of skeleton surfaces and curves, which is not desirable for representing tubular objects. In order to avoid skeleton 
surfaces and extract only the points on the centerline of an object in its elongated direction, we first employ the gradient vector flow (GVF) technique and then apply eigenanalysis of the Hessian matrix to remove false positive points. Finally, we present the results of experiments using CLSM (Confocal Laser Scanning Microscopy) images of blood fibrins and CT images of a skull and lungs. Without loss of generality, our method assumes that the objects of interest are brighter (or darker) than the background and their centerlines coincide with the height ridges on the intensity profile.

\section{Previous Work}

Traditionally, skeletons are obtained by defining a medialness measure and assigning to each point inside an object the distance to the nearest boundary of the object according to the metric defined. Then, on the centerline of the object are the points of equi-distance from at least two object boundary points, forming a ridge on the distance surface. A skeleton is the projection of the ridge onto the image plane. This method, however, cannot be applied directly to grayscale images, where the object's boundary is not known a priori. In grayscale images, including medical images, the objects of interest are usually identified as relatively brighter (or darker) regions and intensity ridges tend to be at the center of such regions at a given scale. Therefore, intensity ridges have been used as a reliable approximation to the skeletons $4 \underline{6}$ ].

Previous work on skeletonization can be classified into two categories. The first approach consists of extracting ridge points and connecting them in a postprocessing step [7/91216. Since it usually uses purely local criteria, this approach generates false positives for ridge points. To obtain skeletons as connected curves, it has to use elaborate grouping schemes [5].

The second approach extracts skeletons by searching for a ridge point at the neighborhood of a given ridge point 12 10 18. This approach generates skeletons as curves without any post-processing steps and the thinness of the skeletons is guaranteed. However, searching extrema in high dimensional parameter space can be computationally expensive. It also needs to provide a set of criteria or heuristics to handle special cases such as branching points and endpoints. Finally, the seed points selection, which is usually done manually, may hinder the method from automation.

In this paper, we propose an efficient method for ridge point detection. It is a gradient-based method especially for objects with almost circular crosssections. Using the gradients of distance maps or intensity profiles usually generates skeleton surfaces for 3D objects, which is not desirable for representing tubular objects. To extract only the points on the centerline along the object's elongated direction, we also employ the gradient vector flow (GVF) technique and eigenanalysis of the Hessian matrix. 


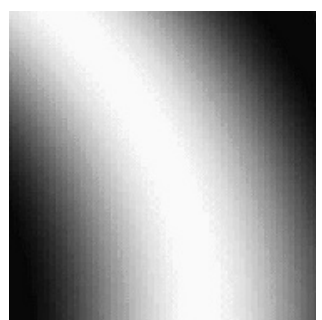

(a)

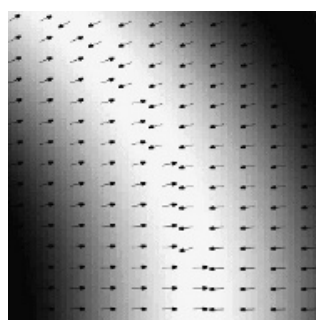

(b)

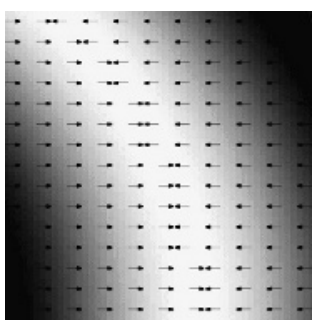

(c)

Fig. 1. Sign barriers. (a) Curvilinear object, (b) Normalized gradient vectors, and (c) Normalized gradient vectors projected onto horizontal lines.

\section{Method}

Scan-conversion algorithm is a gradient-based ridge point detection method. To introduce the algorithm, we begin with the extraction of center lines of curvilinear objects in $2 \mathrm{D}$ and then extend it to 3D. Without loss of generality, we assume that the objects of interest are brighter than the background and their centerlines coincide with the height ridges on the intensity profile.

\subsection{Scan-Conversion Algorithm in 2D}

It is well known that the gradient at any point on such objects generally points towards the ridge and reverses its direction as it crosses the ridge 1112. Similarly, for a point to be on a ridge, it must be a local maximum on some direction, i.e., on a line passing through the point. Consider a line $\left(L_{\theta}\right)$ with an arbitrary orientation $\theta$ and three contiguous points $\left(p_{-1}, p_{0}, p_{+1}\right)$ on it. If $p_{0}$ is on a ridge, the gradients at $p_{-1}$ and $p_{+1}$ must point toward $p_{0}$, forming a sign barrier between them. Figure 1 (b) illustrates an example of sign barriers around a ridge of a curvilinear structure in (a). Note that the sign barriers are not easily identifiable at some part of the object. We can enhance the sign barriers by projecting gradient vectors onto $L_{\theta}$, as illustrated in (c), where $\theta=0$ (horizontal lines) is used. In summary, if a ridge intersects $L_{\theta}$, it generates a sign barrier on the line, i.e., between the two adjacent points that enclose the point of intersection.

It has been reported that examining four orientations $(0,45,90$, and 135 degrees) at each point identifies (in fact, over-identifies) all the ridge points 9 , 14,15]. To determine the minimum number of orientations, consider $L_{\theta}$ again. As discussed before, if a ridge intersects $L_{\theta}$, it generates a sign barrier between two adjacent points enclosing the point of intersection. The ridge point (i.e., the intersection point) is thus guaranteed to be detected by the sign barrier. On the other hand, if a ridge is nearly parallel to $L_{\theta}$ and does not intersect it, the ridge may or may not produce a sign barrier on $L_{\theta}$. Another line $\left(L_{\theta^{\prime}}\right)$ with an orientation substantially different from the orientation of the ridge (or, equivalently, from that of $L_{\theta}$ ) will detect such a ridge point, since the ridge parallel to $L_{\theta}$ will appear perpendicular to $L_{\theta^{\prime}}$ and intersect it at some point, generating a sign barrier on it. For $L_{\theta}$ and $L_{\theta^{\prime}}$ to have sufficiently different 


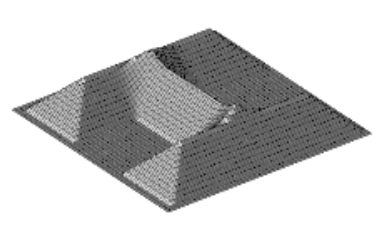

(a)

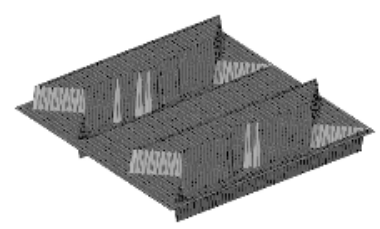

(b)

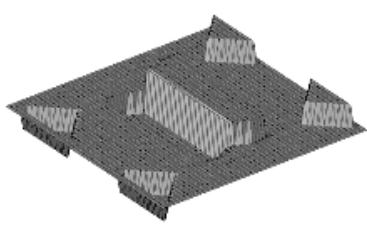

(c)

Fig. 2. Ridge points on $\mathbf{H}$-shaped object. (a) Distance map of the shape, (b) Sign barriers on $L_{0}$, and (c) Sign barriers on $L_{90}$.

orientation, it is natural to use $\theta^{\prime}=\theta+90$. Figure 2 succinctly illustrates these observations. Figure 2(a) shows the distance map of a simple $\mathbf{H}$-shaped object. Although the distance map was used here to generate the gradient vector field of the binary object, we will show how the algorithm can be applied to grayscale images without distance transform in Sect. 3.2. Figure 2(b) and (c) show the sign barriers on $L_{0}$ and $L_{90}$, respectively. Note that the horizontal ridge in the middle of the object is not present in (b), since the ridge is parallel to $L_{0}$ and does not intersect it. As expected, however, the horizontal ridge is detected by $L_{90}$ in (c). Similarly, two vertical ridges of the shape are not detected in (c) but are present in (b). Combining together, $L_{0}$ and $L_{90}$ detect all the ridge points on the distance map.

The discussion so far leads us to a simple scan-conversion algorithm for ridge point detection. Given a gradient vector field $\nabla I(x, y)=\left\langle v_{x}, v_{y}\right\rangle$, two vector fields $\left(N_{\theta}\right.$ and $\left.N_{\theta+90}\right)$ are computed by projecting the gradients onto $L_{\theta}$ and $L_{\theta+90}$. If we use $\theta=0$, the projection is simply:

$$
\begin{aligned}
N_{0}(x, y) & =\left\langle\operatorname{sign}\left(v_{x}\right), 0\right\rangle \\
N_{90}(x, y) & =\left\langle 0, \operatorname{sign}\left(v_{y}\right)\right\rangle .
\end{aligned}
$$

Then, the algorithm scans $N_{0}$ with $L_{0}$ from top to bottom and $N_{90}$ with $L_{90}$ from left to right. For each scan, it searches for sign barriers on $L_{0}$ from left to right and $L_{90}$ from top to bottom. Finally, it combines the points of sign barriers on $L_{0}$ and $L_{90}$.

\subsection{D Extension}

The generalization of scan-conversion algorithm to 3D objects can be achieved by adding another orthogonal line. We use three orthogonal lines, $L_{x}, L_{y}$, and $L_{z}$, parallel to $x, y$, and $z$-axis, respectively. This simple extension of our algorithm, however, produces skeleton surfaces as illustrated in Fig. 3 (a). Three vector fields $\left(N_{x}, N_{y}, N_{z}\right)$ were generated from the distance map gradients of a parallelepiped.

Although skeleton surfaces from 3D objects are theoretically correct $[3$, we are more interested in skeleton curves especially for tubular objects, i.e., the centerlines along the objects' elongated direction. The difficulty in using distance maps for extraction of skeleton curves of 3D objects is that the distance 


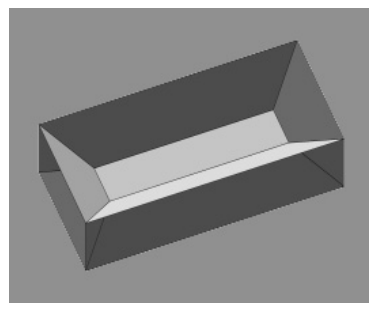

(a)

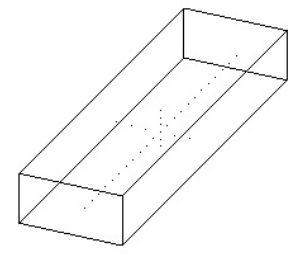

(b)

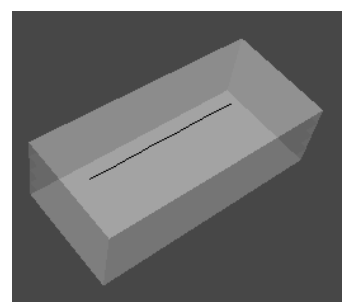

(c)

Fig. 3. Scan-conversion algorithm on a parallelepiped. (a) Skeleton surfaces from distance map. (b) Skeleton points from GVF field. (c) Skeleton curve after pruning by eigenanalysis.

transform generates plateaus (i.e., gradients vanishes there) which constitute skeleton surfaces. In addition, the distance transform cannot be applied directly to grayscale images. To avoid both difficulties, we use the Gradient Vector Flow $(G V F)$ field [17].

The GVF field, $\mathbf{v}(x, y, z)=\langle u(x, y, z), v(x, y, z), w(x, y, z)\rangle$, is defined in 17 to minimize the energy functional

$$
\mathcal{E}=\iiint \mu|\nabla \mathbf{v}|^{2}+|\nabla f|^{2}|\mathbf{v}-\nabla f|^{2} d x d y d z
$$

where the edge map, $f(x, y, z)$, is defined as

$$
f(x, y, z)=-\left|\nabla\left[G_{\sigma}(x, y, z) * I(x, y, z)\right]\right|^{2} .
$$

Note that the edge map $f$ is negated, compared to the original edge map in [17, to make $\nabla f$ point away from its edges. This variational formulation keeps the vector field nearly equal to $\nabla f$ in the regions where $|\nabla f|$ is large (second term) and forces it to be slowly-varying in homogeneous region where $|\nabla f|$ is small (first term). Using the calculus of variations and introducing time to $\mathbf{v}$ $(=\mathbf{v}(\mathbf{x}, t))$, we obtain the following vector diffusion equation:

$$
\begin{aligned}
& \mathbf{v}_{t}=\mu \nabla \mathbf{v}-(\mathbf{v}-\nabla f)|\nabla f|^{2} \\
& \mathbf{v}(\mathbf{x}, 0)=\nabla f(\mathbf{x})
\end{aligned}
$$

where $\mathbf{v}_{t}$ is the partial derivative of $\mathbf{v}$ with respect to time $t$. Since GVF solves these decoupled equations as separate partial differential equations in each component of $\mathbf{v}$, some points that are not on centerlines along the object's elongated direction may have false sign barriers and be detected as shown in Fig. 3 (b). These points are pruned out by eigenanalysis of the Hessian matrix as in [213. The Hessian matrix of a $3 \mathrm{D}$ image, $I(\mathbf{x})$, where $\mathbf{x}=(x, y, z)$ is given by

$$
H=\left[\begin{array}{lll}
I_{x x}^{*}(\mathbf{x} ; \sigma) & I_{x y}^{*}(\mathbf{x} ; \sigma) & I_{x z}^{*}(\mathbf{x} ; \sigma) \\
I_{y x}^{*}(\mathbf{x} ; \sigma) & I_{y y}^{*}(\mathbf{x} ; \sigma) & I_{y z}^{*}(\mathbf{x} ; \sigma) \\
I_{z x}^{*}(\mathbf{x} ; \sigma) & I_{z y}^{*}(\mathbf{x} ; \sigma) & I_{z z}^{*}(\mathbf{x} ; \sigma)
\end{array}\right]
$$




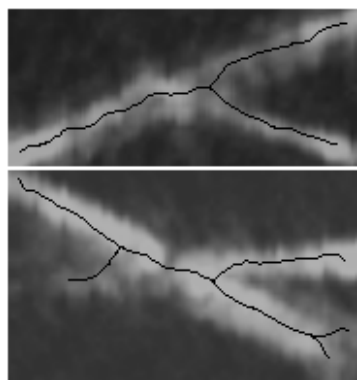

(a)

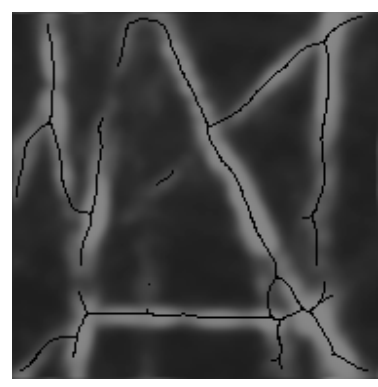

(b)

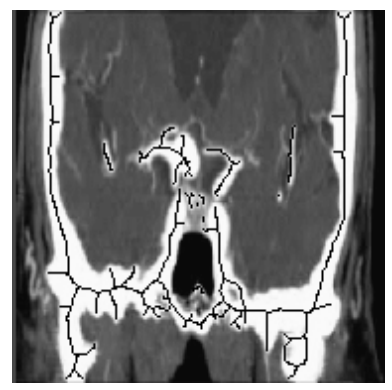

(c)

Fig. 4. (a), (b) CLSM images of blood fibrin. (b) CT image of a skull.

where, $I^{*}=G_{\sigma}(\mathbf{x}) * I(\mathbf{x})$ and the subscripts are the usual partial derivatives. Let $\lambda_{1}(\mathbf{x}), \lambda_{2}(\mathbf{x})$, and $\lambda_{3}(\mathbf{x})\left(\lambda_{1}(\mathbf{x})>\lambda_{2}(\mathbf{x})>\lambda_{3}(\mathbf{x})\right)$ be the eigenvalues of $H$, and $\boldsymbol{e}_{\mathbf{1}}(\mathrm{x}), \boldsymbol{e}_{\mathbf{2}}(\mathrm{x})$, and $\boldsymbol{e}_{\mathbf{3}}(\mathrm{x})$ the corresponding eigenvectors. Then, $\boldsymbol{e}_{\mathbf{1}}(\mathrm{x})$ gives the direction along which the second derivative is maximal and $\lambda_{1}(\mathbf{x})$ the maximum value of the second derivative. At a ridge point $\mathbf{x}, \lambda_{2}(\mathbf{x})$ and $\lambda_{3}(\mathbf{x})$ have the minimum values and must be negative:

$$
\lambda_{3}(\mathbf{x}) \leq \lambda_{2}(\mathbf{x})<0 .
$$

Also, the ridge point $\mathbf{x}$ must be a local maximum on the plane defined by $\boldsymbol{e}_{\mathbf{2}}(\mathbf{x})$ and $e_{3}(\mathrm{x})$, thus:

$$
\boldsymbol{e}_{\mathbf{2}}(\mathbf{x}) \bullet \nabla I^{*}(\mathbf{x} ; \sigma)=0 \text { and } \boldsymbol{e}_{\mathbf{3}}(\mathbf{x}) \bullet \nabla I^{*}(\mathbf{x} ; \sigma)=0 .
$$

Ridge points detected from the GVF field are checked against these conditions and discarded if they fail. Figure 3 (c) shows the result after checking the two conditions on the points in (b).

\section{Experiments}

We evaluate the method using CLSM images of blood fibrins, a CT image of a skull, and CT images of lungs. All experiments were performed on a Dell computer with $2 \mathrm{GHz}$ Xeon processor and $768 \mathrm{MB}$ of memory.

Figure 4 shows the extracted centerlines of curvilinear structures in 2D grayscale images. The scan-conversion algorithm with GVF field eliminates the use of distance maps and can be applied directly to grayscale images. In the figure, 30 iterations of GVF computations were performed with $\mu=0.2$ and $\Delta t=0.01$.

Figure 5 shows the lung vessels extracted from CT data. In the figures, CT images were digitally resliced to make cubic voxels and the volumes of interest (VOI) extracted $-128 \times 128 \times 270,100 \times 128 \times 270$, and $74 \times 120 \times 200$ voxels from left to right in the figure. Then, 50 iterations of GVF computation were performed on each VOI with $\mu=0.2$ and $\Delta t=0.01$. Finally, vessels were 

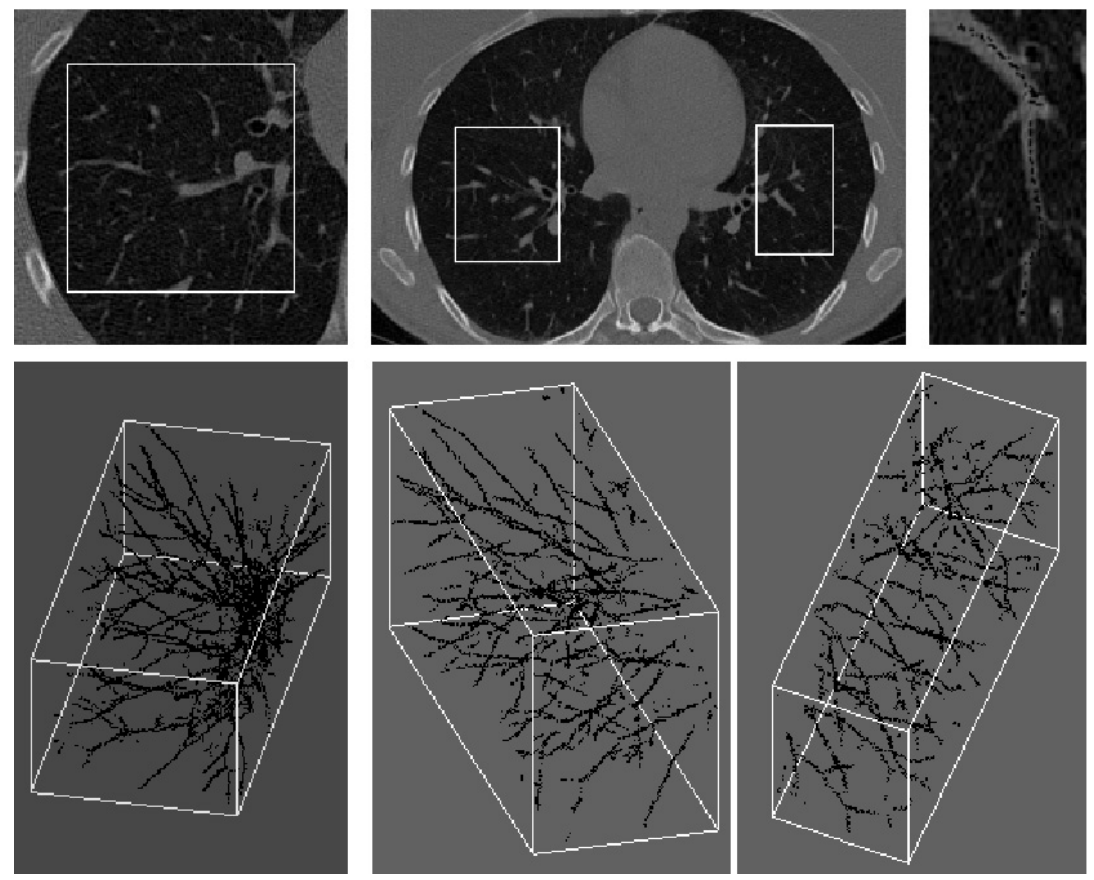

Fig. 5. Lung vessels from CT. The volumes of interest are, from left to right, $128 \times$ $128 \times 270,100 \times 128 \times 270$, and $74 \times 120 \times 200$ voxels.

extracted with the scan-conversion algorithm. The process for each VOI took approximately $3.4 \mathrm{~min}, 1.6 \mathrm{~min}$, and $0.75 \mathrm{~min}$. Note that, due to its extensive floating point calculations, most of the processing times were spent on GVF computation - $3.2 \mathrm{~min}, 1.5 \mathrm{~min}$, and $0.7 \mathrm{~min}$, respectively. However, the scanning process was very fast and took substantially less than a minute for each VOI.

To validate our method, the detected points were projected onto the CT image as shown in the top-right image in Fig. 5. Through visual inspection of the projections, we found the good alignment between the detected points and the centerlines of the vessels.

\section{Conclusion}

We have introduced a scan-conversion algorithm for ridge point detection. The algorithm is a gradient-based method that detects sign barriers on two (or, three in 3D) orthogonal lines.

Gradient vectors are initially obtained from distance maps of given objects, which pose two difficulties. First, the distance transform cannot be applied directly to grayscale images, limiting our applications to binary objects. Second, the distance transform of $3 \mathrm{D}$ objects produces plateaus where gradients vanishes, forcing the algorithm to extract skeleton surfaces. To avoid both difficulties, we 
use the Gradient Vector Flow (GVF) field. Using the GVF field, however, extracts points that are not on the centerline along the object's elongated direction. Such points are identified and discarded by eigenanalysis of the Hessian matrix.

We presented various results of the method using CLSM images of fibrin, skull CT, and 3D lung CT data. Although the GVF computation is demanding, the experiments show that our method is efficient.

\section{References}

1. Aylward, S., Bullitt, E., Pizer, S., Eberly. D.: Intensity Ridge and Widths for Tubular Object Segmentation and Description. MMBIA (1996) 131-138

2. Aylward, S.R., Bullitt, E.: Initialization, Noise, Singularities, and Scale in Height Ridge Traversal for Tubular Object Centerline Extraction. IEEE Trans. Medical Imaging, Vol. 21, no. 2 (2002) 61-75

3. Blum, H.: A Transformation for Extracting New Descriptors of Shape. In: WathenDunn, W (eds.): Models for the Perception of Speech and Visual Form. MIT Press, Cambridge, Mass. (1967) 362-380

4. Eberly, D., Gardner, R., Morse, B., Pizer, S., Scharlach. C.: Ridges for Image Analysis. Journal of Mathematical Imaging and Vision, Vol. 4, (1994) 353-373

5. Fischler, M.A.: The Perception of Linear Structure: A Generic Linker. Image Understanding Workshop (1994) 1565-1579

6. Gauch, J.M., Pizer, S.M.: The Intensity Axis of Symmetry and Its Application to Image Segmentation. PAMI, Vol. 15, no. 8 (1993) 753-770

7. Hancock, E.R., Kittler, J.: Relaxation Refinement of Intensity Ridges. ICPR, Vol. 3 (1992) 459-463

8. Haralick., R.M.: Ridges and Valleys on Digital Images. CVGIP, Vol. 22 (1983) $28-38$

9. Ho, S.B., Dyer, C.R.: Shape Smoothing Using Medial Axis Properies. PAMI, Vol. 8, no. 4 (1986) 512-520

10. López, A.M., Serrat, J.: Tracing Crease Curves by Solving a System of Differential Equations. ECCV (1996) 241-250

11. López, A.M., Lumbreras, F., Serrat, J.: Creaseness from Level Set Extrinsic Curvature. ECCV (1998) 156-169

12. Maintz, J.B., van den Elsen, P.A., Viergever, M.A.: Evaluation of Ridge Seeking Operators for Multimodality Image Matching. PAMI, Vol. 18, no. 4 (1996) 353-365

13. Sato, Y., Nakajima, S., Shiraga, N., Atsumi, H., Yoshida, S., Koller, T., Gerig, G., Kikinis, R.: Three-Dimensional Multi-scale Line for Segmentation and Visualization of Curvilinear Structures in Medical Images. Medical Image Analysis, Vol. 2, no. 2 (1998) 143-168

14. Seemuller, W.W.: The Extraction of Ordered Vector Dranage Networks from Elevation Data. CVGIP, Vol. 47 (1989) 45-58

15. Shih, F.Y., Pu, C.C.: A Skeletonization Algorithm by Maxima Tracking on Euclidean Distance Transform. Pattern Recognition, Vol. 28, no. 3 (1995) 331-341

16. Steger, C.: An Unbiased Detector of Curvilinear Structures. PAMI, Vol. 20, no. 2 (1998) 113-125

17. Xu, C., Prince, J.L.: Snakes, Shapes, and Gradient Vector Flow. IEEE Trans. Image Processing, Vol. 7, no. 3 (1998) 359-369

18. Yim, P.J., Choyke, P.L., Summers, R.M.: Gray-Scale Skeletonization of Small Vessels in Magnetic Resonance Angiography. IEEE Trans. Medical Imaging, Vol. 19, no. 6 (2000) 568-576 